\title{
Infection prophylaxis and management of viral infection
}

\author{
Emily S. Clausen, Lorenzo Zaffiri \\ Department of Pulmonary and Critical Care Medicine, Duke University Medical Center, Durham, NC, USA \\ Contributions: (I) Conception and design: L Zaffiri; (II) Administrative support: None; (III) Provision of study materials or patients: All authors; \\ (IV) Collection and assembly of data: All authors; (V) Data analysis and interpretation: All authors; (VI) Manuscript writing: All authors; (VII) Final \\ approval of manuscript: All authors. \\ Correspondence to: Lorenzo Zaffiri. Department of Pulmonary and Critical Care Medicine, Duke University Medical Center, Durham, NC, USA. \\ Email: lorenzo.zaffiri@duke.edu.
}

\begin{abstract}
Viral infections are associated with significant morbidity and mortality in lung transplant recipients. Importantly, several viral infections have been associated with the development of chronic lung allograft dysfunction (CLAD). Community-acquired respiratory viruses (CARV) such as influenza and respiratory syncytial virus (RSV), are frequently associated with acute and chronic rejection. Cytomegalovirus (CMV) remains a significant burden in regards to morbidity and mortality in lung transplant recipients. Epstein-Barr virus (EBV) is mostly involved with the development of post-transplant lymphoproliferative disorder (PTLD), a lymphoid proliferation that occurs in the setting of immunosuppression. On the other hand, the development of direct acting antivirals for hepatitis $\mathrm{C}$ virus (HCV) is changing the use of HCVpositive organs in transplantation. In this article we will focus on reviewing common viral infections that have a significant impact on lung transplant recipients looking at epidemiology, prevention and potential treatment.
\end{abstract}

Keywords: Viral infections; lung transplant; prophylaxis

Submitted Jun 10, 2019. Accepted for publication Nov 08, 2019.

doi: 10.21037/atm.2019.11.85

View this article at: http://dx.doi.org/10.21037/atm.2019.11.85

\section{Community-acquired respiratory viruses (CARV)}

CARV are common after lung transplantation. In immunocompromised patients these infections can involve the lower respiratory tract and are often associated with significant mortality $(1,2)$. The viral pathogens frequently reported include: influenza A and B, respiratory syncytial virus (RSV), adenovirus, parainfluenza (PIV), human metapneumovirus (hMPV).

CARV infection can occur at any time after lung transplantation, but seasonal variability often occurs. Few cases of donor-derived influenza and adenovirus infection have been reported and associated with significant morbidity $(1,3)$. The most sensitive diagnostic tools available for the detection of CARV infections are nucleic acid amplification assays including polymerase chain reaction (PCR). These assays usually allow for simultaneous detection of a multiple viral pathogens. All patients with a suspected respiratory viral infection should have a nasopharyngeal swab, wash or aspirate for testing $(4,5)$. A prolonged shedding of CARV have described in lung transplant patients, in particular with rhinovirus $(6,7)$. However, the effect of prolonged shedding on the graft is unclear.

In a recent prospective analysis of 98 adult lung transplant recipients, the overall incidence of CARV was 0.76 patient/year. Interestingly, $11.5 \%$ of asymptomatic patients had positive nasopharyngeal swabs compared with $55.4 \%$ positive tests in symptomatic patients. Higher incidences of respiratory viral infection were observed in winter and fall. Picornavirus (rhinovirus and enterovirus), coronaviruses and influenza were the most frequently isolated viral pathogens. While asymptomatic infections were seen mostly with picornaviruses and coronaviruses, infections with Paramyxoviruses and influenza viruses were significantly associated with pneumonia and higher risk of hospitalization (2). Similar findings were reported from a 
prospective Swiss study with overall incidence of respiratory viral infection of 0.83 per patient-year. Respiratory viral infection was detected in $14 \%$ of the screening visit and $34 \%$ of the emergency visits. Picornaviruses were the most commonly identified, while influenza and PIV were associated with $50 \%$ hospitalization rates.

Outcomes associated with respiratory viral infections including acute cellular rejection (ACR) and development of CLAD have been studied in the literature. Often within few months after a BAL-positive respiratory viral infection, lung transplant recipients developed biopsy-proven ACR or decline for FEV1 greater or equal to $20 \%$ compared to recipients without viral isolation (5). However, there are contrasting reports regarding the development of ACR following respiratory viral infection. Despite the Swiss prospective study failed in finding a temporal association between viral infection and ACR (8), in nested case-control analysis from a Spanish group, the presence of respiratory viral infection within the previous 3 months was associated with the development of ACR (2). In a retrospective study including 250 LTR patients, symptomatic CARV infections was independently associated with development of CLAD. The association was stronger in the first months after respiratory viral infections but it was present when all follow-up time was measured (9).

\section{RSV}

RSV is one of the most commonly isolated CARV with incidence up to $16 \%$ after LTR. RSV is transmitted via droplet secretions. The incidence of RSV usually follows community outbreaks with peak incidence from September to April. However, hospital outbreaks have been described as well. The overall mortality for RSV among immunocompromised patients can be as high as $20 \%$. Moreover, in LTR the overall mortality for RSV infections ranges from $10 \%$ to $20 \%$.

RSV infection is associated with significant morbidity and mortality. Acutely, RSV infection is associated with development of bronchiolitis, lower respiratory infection and respiratory failure. A persistent decline greater than $20 \%$ of FEV1 suggestive of development of bronchiolitis obliterans syndrome (BOS) had been described following RSV isolation in lung transplant recipients (10). RSV is therefore considered a distinctive risk factor for the development of CLAD $(11,12)$. Moreover, mortality rates range between $10 \%$ and $20 \%$ after RSV infection in lung transplant recipients $(11,13)$.
To prevent spreading of RSV infections, patients with known or suspected RSV should be isolated from other patients using standard contact precautions. There is no approved vaccine for the prevention of RSV. Recently a humanized RSV-specific monoclonal antibody palivizumab demonstrated to be effective for high risk infants and children with specific underlying clinical conditions. However, no studies have been completed to assess the use of palivizumab in the setting of solid organ transplant (SOT). Due to lack of randomized clinical trials, the treatment of RSV infection is still matter of debate. Primarily, therapy for RSV infection is supportive. Several case series have described therapeutic regimens including ribavirin aerosolized, intravenous (IV) or oral with or without IV immunoglobulin and steroid. Oral ribavirin is the most frequently used due to lower cost compared to aerosolized form despite limited studies comparing the efficacy and safety in the management of RSV infections. In a recent single-center study involving 46 patients (of which 22 lung transplant recipients) showed no differences in mortality, length of stay and resolution of symptoms but an enormous cost avoidance attributable to use of oral ribavirin (14). In two placebo-controlled trials involving lung transplant recipients AL-RSV01, which is a small interfering RNA (siRNA) that acts preventing viral replication, had been studied. In the first trial the nebulized ALN-RSV01 was administered to 24 lung transplant recipients with RSV infection for 3 days demonstrating safety and tolerability. Moreover, new or progressive FEV1 decline consistent with BOS at 90 day was decreased compared with placebo (15). Similarly, in the subsequent phase $2 \mathrm{~b}$ study nebulized ALNRSV01 was well tolerated for 5 days and demonstrated a significant treatment effect when initiated early from symptoms onset on the development of BOS on day 180 compared with placebo (16).

\section{Influenza}

Influenza virus A and B belong to the virus family Orthomyxoviridae and are able to cause a contagious respiratory disease in humans (17). Influenza A viruses generally surface from a zoonotic reservoir before spreading among human. On the other hand, influenza B virus is almost exclusively found in human host. Influenza A viruses in particular present two peculiar antigenic properties that allow to evade host responses and perpetuates seasonal epidemics and pandemic. Minor changes induced by the point mutations that usually involve the hemagglutinin and 
neuraminidase are called antigenic drift. More complex arrangement that allow for the mixing genetic information of different viral strains giving rise to novel and gene-reassorted virus strains is called antigenic shift, which is associated with development of pandemics as in $2009(18,19)$.

Influenza viruses are the cause of annual epidemics of respiratory illness that is associated with significant morbidity and mortality in immunocompetent and immunosuppressed population. The annual outbreaks have a seasonal distribution that mostly occur during winter months. Interestingly, there is still a limited understanding on influenza transmission, but the generally accepted way of transmission are direct contact from infected patients, droplets and aerosols (20). The cumulative incidence of influenza virus infection in SOT recipients is estimated to be up to $13 \%$ (4). According to a single-center retrospective study evaluating in 33 immunosuppressed patients, 39\% of all respiratory infections were caused by influenza viruses (21) Influenza virus infection can present mild respiratory complains but it is often associated with progression to lower respiratory tract infection, bacterial superinfection, respiratory failure and later with the development of chronic rejection $(22,23)$. Severe influenza disease is usually observed in early post-transplant periods likely due to more intense immunosuppression. Mortality ranges between $2-4 \%$ but it can increase up to $21 \%$ in lung transplant recipients with pre-existing grade 3 BOS during the recent H1N1 pandemic (24). Several tests are available for the diagnosis of influenza including rapid antigen detection, direct fluorescent antibodies (DFA), but PCR assay is considered the gold standard for diagnosis.

In order to prevent the spread of influenza viruses is recommended to use appropriate droplet precautions and vaccination of recipients and their close contacts. Annual vaccination is recommended to all lung transplant recipients (25). Influenza vaccine should be administered after the first 3 months from transplantation or intensified immunosuppression following treatment of rejection. Vaccine can be given as early as 1 month after transplantation in case of high influenza activities or pandemic (26). There is different type of vaccine: trivalent vaccine, containing two strains of influenza $A$ and one strain of influenza $\mathrm{B}$, and a quadrivalent vaccine that comprises two strains of each influenza virus. Responses in terms of immunogenicity are variable in all SOT recipients, mostly affected by type of transplanted organ and immunosuppression regimens and intensity. Mycophenolate mofetil appears to be associated with poorer antibody response, while contrasting data are available for other antirejection drugs (27). In a large multicenter prospective studies including 616 transplant patients, of which 116 lung transplant recipients, influenza vaccination was associated with decrease disease severity (28). There are some concerns that vaccination might be associated with development of de novo DSA and/or allograft rejection. The evidence is poor and a recent systematic review on vaccine safety provided reassurance on the safety of vaccination in SOT recipients (29).

There are only few retrospective studies including lung transplant patients of the use of oseltamivir for the treatment of influenza infection $(24,30)$. Therefore, there are no recommendation on the optimal timing, dose and duration in lung transplant recipients with confirmed influenza infection. However, it has been suggested that antiviral therapy should be given to all lung transplant recipients with suspected or confirmed influenza infection despite severity or onset of symptoms. Oseltamivir is generally well-tolerated and has shown to improve outcomes particularly if initiated within 48 hours from symptoms onset (24). Recently, a new antiviral drug, baloxavir marboxil, had been approved for the treatment of influenza A and B (31). Studies in SOT recipients are currently not available.

\section{Adenovirus}

Adenoviruses are a widespread group of viruses with over 60 serotypes known to cause a variety infections including respiratory, gastrointestinal and febrile disease in immunocompetent hosts (32). Adenoviruses are divided in seven species (from A to $G$ ) depending on several viral characteristics (33). The incidence of adenovirus among lung transplant recipients or for that matter all SOTs is not well-defined. More data exists for bone marrow transplant populations with estimates a cumulative incidence of $3 \%$ in adult bone marrow transplant recipients, with those having an allogeneic versus an autologous transplant having disproportionately higher risk (34).

Among pediatric lung transplant recipients, a single center has reported a cumulative incidence of $7 \%$ for adenovirus pneumonia (35) while a cumulative incidence of $2.5 \%$ was observed in an adult cohort (13). Adenovirus infection can be acquired de novo but in most of adult SOT recipients it manifest as reactivation of a latent infection of the recipient or from the graft itself. In immunocompromised patients, endogenous reactivation 
of adenovirus seems to be the predominant cause of disease based on studies demonstrating identical strain of adenovirus isolated prior and post-transplant in allogeneic hematopoietic stem cell transplant recipients (36). Usually, the primary site of adenovirus disease is the transplanted graft with manifestations including necrotizing pneumonias, nephritis, hemorrhagic cystitis and disseminated disease $(37,38)$. With regards to outcomes, adenovirus infection in lung transplant recipients has been associated with graft failure, particularly with FEV1 decline consistent with BOS (3). Mortality from adenovirus infection has been reported in both pediatric and lung transplant populations $(13,35)$.

Multiple diagnostic tests exist for adenovirus but realtime PCR assays are the recommended standard and can be used for detection in most specimen types $(39,40)$. However, these results should be correlated with clinical presentation and histopathology in order to distinguish asymptomatic infection, adenovirus disease and disseminated disease. This recommendation derives from the fact that asymptomatic patients can shed for prolonged periods of time. Despite the lack of general consensus, the American Society of Transplantation has recommended to define an asymptomatic adenovirus infection as the detection of adenovirus from patient samples (blood, urine, stools, BAL) in absence of signs or symptoms. While the detection of adenovirus in biopsy specimen or from BAL along with the presence signs or symptoms of organ involvement should be considered as adenovirus disease. Finally, a disseminated infection is characterized by the involvement of 2 or more organs not including viremia $(33,38)$. With regards to prevention of adenovirus, there are no vaccines or standard prophylaxis regimens available in hospital settings, strict droplet and contact precautions are recommended for those that test positive for adenovirus.

Similar to immunocompetent hosts, treatment of adenovirus infection in lung transplant recipient starts with supportive care. If possible, reduction in immunosuppressive therapy is recommended to aid with clearance (39). Potential antiviral agents against adenovirus include ribavirin and cidofovir. Use of IV ribavirin for adenovirus infection in bone marrow transplant patients has not shown any clear benefit (34) and is not recommended (39). There has been some success with the use of IV cidofovir but usage is significantly limited by nephrotoxicity (35). Brincidofovir is an oral, lipid derivative of cidofovir that has shown acceptable tolerability, safety, and efficacy in bone marrow transplant recipients $(41,42)$. Further research of the utility of brincidofovir among other SOT is ongoing.

It is worth to mention that other respiratory viruses are often identified in lung transplant recipients and are linked with development of decline of lung function consistent with CLAD $(43,44)$. The after lung transplant, a cumulative incidence of $5-7 \%$ have been described for PIV virus and hMPV (2). Currently, only supportive therapy is recommended, due to the lack of vaccines or approved antiviral drugs with clinical benefits.

\section{Cytomegalovirus (CMV)}

The cumulative incidence of CMV disease among lung transplant recipients has remained at $20 \%$ to $50 \%$ with some reports up to $80 \%$, even in the era of preventive prophylactic strategies (45-47). Active CMV infection manifests as fever, bone marrow suppression, and tissue invasive disease including pneumonitis and colitis. Aside from direct effects of CMV infection itself, CMV has long been associated with increased acute rejection episodes (48) and FEV1 decline consistent with BOS $(49,50)$. Donor (D) and recipient (R) mismatch, specifically CMV D+Rrecipient are at significantly higher risk of developing $\mathrm{CMV}$ disease (51). Along with increased risk of CMV disease, CMV D+R- recipients have higher overall mortality than CMV D-R- for lung transplant recipients (52).

Given the serious implications of CMV disease, most lung transplant centers practice universal prophylaxis in the initial months post-transplant regardless of CMV status, with valganciclovir being an effective oral regimen (53). Optimal duration of prophylaxis has been the subject of much study, with evidence supporting extending duration of prophylaxis to minimum of 1 year post-transplant, particularly for CMV D+R- recipients $(54,55)$. Use of CMV prophylaxis after treatment of rejection with antilymphocyte antibodies is also recommended. Hematologic adverse events related to valganciclovir are not uncommon and newer alternative oral regimens are becoming more available. Letermovir has been shown effective in stem cell transplant populations and could be considered an off-label alternative for prophylaxis that is not myelosuppressive (56). The role of CMV immunoglobulin in post-transplant prophylaxis is not well-supported by what evidence exists (57) though it is still in use as an adjunct therapy for CMV disease treatment at some centers (58).

Recommended treatment regimens for CMV disease with normal renal function include oral valganciclovir $900 \mathrm{mg}$ twice daily and intravenous (IV) ganciclovir $5 \mathrm{mg} / \mathrm{kg} / \mathrm{dose}$ 
twice daily (59). For any CMV disease that is tissue invasive or considered clinically severe, IV ganciclovir is recommended first-line over oral valganciclovir. Treatment response is monitored with once weekly quantitative $\mathrm{CMV}$ nucleic acid amplification. For those that do not also have evidence of rejection, decreasing immunosuppression regimens from 3 to 2 drugs during the duration of $\mathrm{CMV}$ treatment may result in sooner disease resolution (60).

Incidence of ganciclovir resistant $\mathrm{CMV}$ have been reported at $6-11.9 \%$ among lung transplant recipients in prior decades but likely is higher in the current era with the increase in use of CMV prophylaxis, increase in number of lung transplants, and increase in use of induction agents at time of transplant $(61,62)$. Transplant recipients with recurrent $\mathrm{CMV}$ infection or persistent $\mathrm{CMV}$ on prolonged therapy should undergo testing, particularly for UL97 and UL54 gene mutations (59). An option for ganciclovir resistance is IV foscarnet which at times is also given in combination with ganciclovir, particularly if there is also cidofovir resistance present. Cidofovir can be considered as salvage therapy in strains with both foscarnet and ganciclovir resistance but there are limits with nephrotoxicity and possible rapid development resistance. Maribavir is a UL97 inhibitor that has shown activity against CMV strains resistant to ganciclovir and foscarnet (63). A phase II study of Maribavir $400 \mathrm{mg}$ twice daily showed effectiveness against resistant and refractory CMV infection in hematopoietic cell transplant recipients (64) and is undergoing further study among SOT populations. Improvements in testing and treatments of $\mathrm{CMV}$ resistance continue to evolve but guidelines are based upon expert consensus statements.

\section{Epstein-Barr virus (EBV)}

EBV is widely disseminated herpesvirus that is transmitted by contact with oral secretions. Approximately $90 \%$ of adults worldwide have antibodies to EBV and the majority of the primary infections are asymptomatic. In adolescent and adults, EBV is primary agent of infectious mononucleosis but it is also associated with several malignancies including B and T cell lymphomas, Hodgkin's lymphoma, gastric carcinoma and nasopharyngeal carcinoma (NPC).

Following the initial infection, EBV utilizes different programs of gene expression to establish a quiescent but persistent infection of B cells. In immunocompetent hosts, these events are constantly monitored by the immune system: active viral replication induces NK cell activation, production of serum antibodies to different EBV proteins and a large expansion of cytotoxic CD8+ T lymphocytes $(65,66)$. In the setting of immunosuppression, likely due to blunting of these immune responses, EBV is associated in $70-80 \%$ of the cases with the development of posttransplant lymphoproliferative disorder (PTLD).

The development of an adaptive $\mathrm{T}$-cell immune response is central to controlling EBV-infected B cell proliferation and virus replication. Despite high variability in the EBVspecific CD8+ T cell counts, no difference was noted among patients with PTLD when compared to SOT patients with EBV reactivation or healthy controls (67). Studies by Martinez and colleagues have shown that pediatric transplant patients are able to mount a primary T-cell response to EBV. However, the magnitude of the response to EBV lytic and latent proteins by polyfunctional $\mathrm{T}$ cell response is attenuated when compared to healthy subjects $(68,69)$ Collectively all these prior studies illustrate that T-cell immune responses to EBV vary post-transplant and may favor the development of PTLD.

PTLD presents as a clinically and pathologically heterogeneous group of lymphoproliferative diseases. Data from international transplant registries suggest that the overall prevalence of EBV-associated PTLD ranges between $1-20 \%$ after SOT $(70,71)$. In adult lung transplant recipients a cumulative incidence up to $10 \%$ of PTLD had been observed, higher than most other commonly transplanted organs $(72,73)$. Despite the low incidence, PTLD represents one of the most serious complications in SOT recipients. Mortality after PTLD in lung transplant is up to $50 \%$ with recipients dying due to treatment failures or complications of chemotherapy (74-76). Based on small studies, the risk of developing PTLD is highest in the first year after transplant. However, a second peak of PTLD is evident many years after transplantation (77). Several efforts have been done to identify the patients who are at risk of developing PTLD. Currently, EBV status mismatch, CMV seronegativity and intense immunosuppression are considered the major risks factors for the development of PTLD (73-75,78). Other associations have also described including certain HLA types and the expression of specific viral genes (79). Regarding treatment, reduction of immunosuppression is cornerstone and the initial step in treating patients with PTLD, in order to reduce the disequilibrium between immunosuppression and immunosurveillance. This approach is often associated with increased risk of graft failure. Rituximab, anti-CD20 
monoclonal antibody, is now considered the standard therapy along with reduction of immunosuppression based on results from several phase 2 trials $(80,81)$. Other chemotherapy regimens, including R-CHOP (rituximab, cyclophosphamide, doxorubicin hydrochloride, vincristine and prednisone), can be used in case of lack of responses to initial management. Recently, trials on EBV-specific cytotoxic $\mathrm{T}$ cells generated from blood donors have shown interesting results in patients with PTLD who failed conventional treatments $(82,83)$.

Prevention of the development of PTLD depends on limiting the immunosuppression. Routine EBV PCR to monitor the presence of EBV viremia have been suggested especially in lung transplant recipients with negative EBV serostatus. There are some caveats. First, although a PCR is a standard and reliable method to measure EBV loads, there are no standard protocols, kits, or machines. Individual centers and laboratories will develop their own cutoff values. Therefore, the World Health Organization (WHO) International Standard for EBV was developed based on the results of a worldwide collaborative study group, and was released for the standardization of quantitative PCR (84). Secondly, there is no consensus on monitoring EBV loads in plasma versus whole blood. Immunocompetent healthy EBV-carriers have a measurable EBV DNA in their whole blood, but EBV DNA is hardly detectable in plasma. Similarly, EBV loads measure in whole blood were higher compared to plasma samples from 10 PTLD patients as described by Wagner et al. (85). Currently, there are no clear recommendations to monitor EBV viral loads in SOT.

The role of antiviral agents is equivocal. Valganciclovir or ganciclovir, used for CMV prophylaxis, are able to block EBV replication in vitro. While acyclovir acts in the lytic phase of EBV replication but has no effect on reactivation of latent virus as observed in malignancies. Evidence of a beneficial effect of ganciclovir comes from a retrospective studies of pediatric liver recipients. In this study 18 patient considered at high-risk of developing PTLD due to EBV mismatch (donor EBV positive/recipient EBV negative) received intravenous ganciclovir, 22 low-risk patients (EBV recipients positive) received intravenous ganciclovir during hospitalization followed by acyclovir. There were no cases of PTLD in the high-risk liver transplant patients, and 2 cases of PTLD in the low-risk which resolved with reduction of immunosuppression (86). A different approach using preemptive rituximab has been described in hematopoietic stem cell transplants presenting with detectable EBV PCR.
This strategy appeared to be safe and effective in preventing the development of PTLD (87). There are no studies in lung transplant recipients to explore this option yet.

\section{Other herpesviruses}

Herpes simplex virus type 1 (HSV-1) and type 2 (HSV-2) are common infections in immunocompetent hosts and are linked to reactivation after organ transplantation. HSV1 present a prevalence in the general population of $70-90 \%$ while HSV is approximately $20 \%$ (88). Clinically, HSV viruses reactivation include oral and genital mucocutaneous vesicular rash. Rarely, severe cases of disseminated HSV disease can be observed in SOT recipients (89). The antiviral prophylaxis given for CMV prophylaxis is including ganciclovir, acyclovir or valacyclovir is also effective in preventing HSV reactivation. Treatment of HSV reactivation consists of oral acyclovir (90).

Varicella-zoster virus (VZV) is associated with the development of two clinically distinct diseases: varicella and herpes-zoster (HZ) or shingles. VZV presents a seroprevalence close to $100 \%$ in Europe and North America. Interestingly the incidence of $\mathrm{HZ}$ is growing in immunocompetent hosts, with a median incidence of 4-4.5 per 1,000 person-year (91). Due to the immunosuppression and decreased $\mathrm{T}$ cell immunity, SOT recipients are at increased risks of developing $\mathrm{HZ}$ (92). HZ can be observed in SOT recipients and in particular in lung transplant with a cumulative probability up to $20 \%$ (92). VZV reactivation usually occurs after the use of antiviral prophylaxis is discontinued. Therapy for VZV infection consists of administration of oral acyclovir, valacyclovir. Despite rare, cases of fatal disseminated VZV have been described in lung transplant recipients. IV acyclovir is drug of choice in this case (93). Strategies to attenuate VZV reactivation include: pre-transplant vaccination with live attenuated Oka vaccine as recommended in general elderly population (94). A positive vaccine history or $\mathrm{VZV}$ antibody titers before SOT protect against VZV disease after SOT. Recently, a dead recombinant zoster vaccine was tested in selected group of kidney transplant recipients, demonstrating safety and immunogenicity (95). More studies regarding clinical benefits and lung transplant recipients are still lacking.

\section{Hepatitis virus}

Hepatitis B virus (HBV) and hepatitis C virus (HCV) are cause of acute and chronic hepatitis after transplantation (96). 
However, vaccination and the recent development of direct acting anti-viral drugs have helped in increasing the use of organs from donors with known viral hepatitis.

$\mathrm{HBV}$ is a major cause of chronic hepatitis, cirrhosis and hepatocellular carcinoma worldwide. Its prevalence and associated mortality have started to decrease following effective vaccination. Lung transplant candidate should be screened prior to transplantation (97). Patients who had received $\mathrm{HBV}$ vaccine (anti-HBs IgG positive) and patients who have been previously infected (anti-HBs IgG and anti-HBc IgG positive) are considered candidate for lung transplantation. Patients without immunity should receive HBV vaccination. Accelerated schedules can also be given but may be less immunogenic (94). According to recent guidelines from the ISHLT, chronic HBV infection should be considered a relative contraindication and selected candidates should be screened for presence of cirrhosis or hepatocellular carcinoma (98). Antiviral therapy should be continued indefinitely after transplantation with drugs of choice including entecavir, tenofovir and lamivudine (96).

Similarly, HCV is associated with development of chronic hepatitis, cirrhosis and hepatocellular carcinoma (99). There is no vaccine for the prevention of $\mathrm{HCV}$ transmission. Initial screening for antibody to HCV should be done at the time of transplant assessment. In case of positive HCV serology, further studies including genotyping, liver scan and biopsy are necessary (100). Chronic HCV infection remains an absolute contraindication. However, a recent analysis of the UNOS database showed that HCVseropositive recipients have a similar 5-year survival when compared to HCV-negative (101). In selected cases with absence of cirrhosis and hepatocellular carcinoma lung transplantation may be considered.

The use of HBV and HCV positive donors has been reported in the literature. The risk of $\mathrm{HBV}$ transmission is insignificant in candidate with proven immunity (102). Therefore, efforts should be made to vaccinate prior to transplantation. In HBV non immune patients, protocols to monitor the development of transaminitis, seroconversion or infection are recommended (102). The 5 -year mortality was not significantly different between 333 recipients of anti-HBc positive lungs and heart-lung transplantation and 13,233 recipients of anti-HBc negative organs. Prophylaxis following anti-HBc positive organ transplant with lamivudine is suggested for 12 months in case of positive HBV DNA in the blood (96). Transplantation of HCV-positive organs almost always results in viral transmission. Currently, the use of HCV positive organs is limited to HCV-positive recipients. However, recently the use of $\mathrm{HCV}$-positive organs in $\mathrm{HCV}$-negative recipients is growing due to the newer direct acting anti-viral drugs such sofosbuvir, ledipasvir and velpatasvir which allow eradication of HCV infection (103-105).

\section{Acknowledgments}

Funding: None.

\section{Footnote}

Provenance and Peer Review: This article was commissioned by the Guest Editors (Masaaki Sato and Dong Tian) for the series "Strategies to Achieve Long-Term Success of Lung Transplantation" published in Annals of Translational Medicine. The article was sent for external peer review organized by the Guest Editors and the editorial office.

Conflicts of Interest: The series "Strategies to Achieve LongTerm Success of Lung Transplantation" was commissioned by the editorial office without any funding or sponsorship. The authors have no other conflicts of interest to declare.

Ethical Statement: The authors are accountable for all aspects of the work in ensuring that questions related to the accuracy or integrity of any part of the work are appropriately investigated and resolved.

Open Access Statement: This is an Open Access article distributed in accordance with the Creative Commons Attribution-NonCommercial-NoDerivs 4.0 International License (CC BY-NC-ND 4.0), which permits the noncommercial replication and distribution of the article with the strict proviso that no changes or edits are made and the original work is properly cited (including links to both the formal publication through the relevant DOI and the license). See: https://creativecommons.org/licenses/by-nc-nd/4.0/.

\section{References}

1. Meylan PR, Aubert JD, Kaiser L. Influenza transmission to recipient through lung transplantation. Transpl Infect Dis 2007;9:55-7.

2. Peghin M, Hirsch HH, Len O, et al. Epidemiology and Immediate Indirect Effects of Respiratory Viruses in Lung Transplant Recipients: A 5-Year Prospective Study. Am J Transplant 2017;17:1304-12. 
3. Bridges ND, Spray TL, Collins MH, et al. Adenovirus infection in the lung results in graft failure after lung transplantation. J Thorac Cardiovasc Surg 1998;116:617-23.

4. Ison MG. Respiratory viral infections in transplant recipients. Antivir Ther 2007;12:627-38.

5. Kumar D, Husain S, Chen MH, et al. A prospective molecular surveillance study evaluating the clinical impact of community-acquired respiratory viruses in lung transplant recipients. Transplantation 2010;89:1028-33.

6. de Lima CR, Mirandolli TB, Carneiro LC, et al. Prolonged respiratory viral shedding in transplant patients. Transpl Infect Dis 2014;16:165-9.

7. Kaiser L, Aubert JD, Pache JC, et al. Chronic rhinoviral infection in lung transplant recipients. Am J Respir Crit Care Med 2006;174:1392-9.

8. Bridevaux PO, Aubert JD, Soccal PM, et al. Incidence and outcomes of respiratory viral infections in lung transplant recipients: a prospective study. Thorax 2014;69:32-8.

9. Fisher CE, Preiksaitis CM, Lease ED, et al. Symptomatic Respiratory Virus Infection and Chronic Lung Allograft Dysfunction. Clin Infect Dis 2016;62:313-9.

10. Kumar D, Erdman D, Keshavjee S, et al. Clinical impact of community-acquired respiratory viruses on bronchiolitis obliterans after lung transplant. Am J Transplant 2005;5:2031-6.

11. Khalifah AP, Hachem RR, Chakinala MM, et al. Respiratory viral infections are a distinct risk for bronchiolitis obliterans syndrome and death. Am J Respir Crit Care Med 2004;170:181-7.

12. Gottlieb J, Schulz TF, Welte T, et al. Communityacquired respiratory viral infections in lung transplant recipients: a single season cohort study. Transplantation 2009;87:1530-7.

13. Palmer SM Jr, Henshaw NG, Howell DN, et al. Community respiratory viral infection in adult lung transplant recipients. Chest 1998;113:944-50.

14. Trang TP, Whalen M, Hilts-Horeczko A, et al. Comparative effectiveness of aerosolized versus oral ribavirin for the treatment of respiratory syncytial virus infections: A single-center retrospective cohort study and review of the literature. Transpl Infect Dis 2018;20:e12844.

15. Zamora MR, Budev M, Rolfe M, et al. RNA interference therapy in lung transplant patients infected with respiratory syncytial virus. Am J Respir Crit Care Med 2011;183:531-8.

16. Gottlieb J, Zamora MR, Hodges T, et al. ALN-RSV01 for prevention of bronchiolitis obliterans syndrome after respiratory syncytial virus infection in lung transplant recipients. J Heart Lung Transplant 2016;35:213-21.

17. Krammer F. Emerging influenza viruses and the prospect of a universal influenza virus vaccine. Biotechnol J 2015;10:690-701.

18. Schmolke M, Garcia-Sastre A. Evasion of innate and adaptive immune responses by influenza A virus. Cell Microbiol 2010;12:873-80.

19. García-Sastre A, Schmolke M. Avian influenza A H10N8-a virus on the verge? Lancet 2014;383:676-7.

20. Killingley B, Nguyen-Van-Tam J. Routes of influenza transmission. Influenza Other Respir Viruses 2013;7 Suppl 2:42-51.

21. Garbino J, Soccal PM, Aubert JD, et al. Respiratory viruses in bronchoalveolar lavage: a hospital-based cohort study in adults. Thorax 2009;64:399-404.

22. Garantziotis S, Howell DN, McAdams HP, et al. Influenza pneumonia in lung transplant recipients: clinical features and association with bronchiolitis obliterans syndrome. Chest 2001;119:1277-80.

23. Billings JL, Hertz MI, Savik K, et al. Respiratory viruses and chronic rejection in lung transplant recipients. J Heart Lung Transplant 2002;21:559-66.

24. Kumar D, Michaels MG, Morris MI, et al. Outcomes from pandemic influenza A H1N1 infection in recipients of solid-organ transplants: a multicentre cohort study. Lancet Infect Dis 2010;10:521-6.

25. Rubin LG, Levin MJ, Ljungman P, et al. 2013 IDSA clinical practice guideline for vaccination of the immunocompromised host. Clin Infect Dis 2014;58:309-18.

26. Kumar D, Blumberg EA, Danziger-Isakov L, et al. Influenza vaccination in the organ transplant recipient: review and summary recommendations. Am J Transplant 2011;11:2020-30.

27. Hayney MS, Welter DL, Francois M, et al. Influenza vaccine antibody responses in lung transplant recipients. Prog Transplant 2004;14:346-51.

28. Kumar D, Ferreira VH, Blumberg E, et al. A 5-Year Prospective Multicenter Evaluation of Influenza Infection in Transplant Recipients. Clin Infect Dis 2018;67:1322-9.

29. Mulley WR, Dendle C, Ling JEH, et al. Does vaccination in solid-organ transplant recipients result in adverse immunologic sequelae? A systematic review and metaanalysis. J Heart Lung Transplant 2018;37:844-52 .

30. Fox BD, Raviv Y, Rozengarten D, et al. Pandemic influenza (H1N1): impact on lung transplant recipients and candidates. J Heart Lung Transplant 2010;29:1034-8. 
31. O'Hanlon R, Shaw ML. Baloxavir marboxil: the new influenza drug on the market. Curr Opin Virol 2019;35:14-8.

32. Lion T. Adenovirus infections in immunocompetent and immunocompromised patients. Clin Microbiol Rev 2014;27:441-62.

33. Echavarría M. Adenoviruses in immunocompromised hosts. Clin Microbiol Rev 2008;21:704-15.

34. La Rosa AM, Champlin RE, Mirza N, et al. Adenovirus infections in adult recipients of blood and marrow transplants. Clin Infect Dis 2001;32:871-6.

35. Doan ML, Mallory GB, Kaplan SL, et al. Treatment of adenovirus pneumonia with cidofovir in pediatric lung transplant recipients. J Heart Lung Transplant 2007;26:883-9.

36. Veltrop-Duits LA, van Vreeswijk T, Heemskerk B, et al. High titers of pre-existing adenovirus serotype-specific neutralizing antibodies in the host predict viral reactivation after allogeneic stem cell transplantation in children. Clin Infect Dis 2011;52:1405-13.

37. Ohori NP, Michaels MG, Jaffe R, et al. Adenovirus pneumonia in lung transplant recipients. Hum Pathol 1995;26:1073-9.

38. Florescu DF, Schaenman JM. Adenovirus in solid organ transplant recipients: Guidelines from the American Society of Transplantation Infectious Diseases Community of Practice. Clin Transplant 2019:e13527.

39. Matthes-Martin S, Feuchtinger T, Shaw PJ, et al. European guidelines for diagnosis and treatment of adenovirus infection in leukemia and stem cell transplantation: summary of ECIL-4 (2011). Transpl Infect Dis 2012;14:555-63.

40. Sandkovsky U, Vargas L, Florescu DF. Adenovirus: current epidemiology and emerging approaches to prevention and treatment. Curr Infect Dis Rep 2014;16:416.

41. Grimley MS, Chemaly RF, Englund JA, et al. Brincidofovir for Asymptomatic Adenovirus Viremia in Pediatric and Adult Allogeneic Hematopoietic Cell Transplant Recipients: A Randomized Placebo-Controlled Phase II Trial. Biol Blood Marrow Transplant 2017;23:512-21.

42. Hiwarkar P, Amrolia P, Sivaprakasam P, et al. Brincidofovir is highly efficacious in controlling adenoviremia in pediatric recipients of hematopoietic cell transplant. Blood 2017;129:2033-7.

43. Vilchez RA, Dauber J, McCurry K, et al. Parainfluenza virus infection in adult lung transplant recipients: an emergent clinical syndrome with implications on allograft function. Am J Transplant 2003;3:116-20.
44. Dosanjh A. Respiratory metapneumoviral infection without co-infection in association with acute and chronic lung allograft dysfunction. J Inflamm Res 2015;8:79-82.

45. Mitsani D, Nguyen MH, Kwak EJ, et al. Cytomegalovirus disease among donor-positive/recipient-negative lung transplant recipients in the era of valganciclovir prophylaxis. J Heart Lung Transplant 2010;29:1014-20.

46. Gagermeier JP, Rusinak JD, Lurain NS, et al. Subtherapeutic ganciclovir (GCV) levels and GCVresistant cytomegalovirus in lung transplant recipients. Transpl Infect Dis 2014;16:941-50.

47. Grossi P, Mohacsi P, Szabolcs Z, et al. Cytomegalovirus Immunoglobulin After Thoracic Transplantation: An Overview. Transplantation 2016;100 Suppl 3:S1-4.

48. Roux A, Mourin G, Fastenackels S, et al. CMV driven CD8(+) T-cell activation is associated with acute rejection in lung transplantation. Clin Immunol 2013;148:16-26.

49. Duncan SR, Paradis IL, Yousem SA, et al. Sequelae of cytomegalovirus pulmonary infections in lung allograft recipients. Am Rev Respir Dis 1992;146:1419-25.

50. Snyder LD, Finlen-Copeland CA, Turbyfill WJ, et al. Cytomegalovirus pneumonitis is a risk for bronchiolitis obliterans syndrome in lung transplantation. Am J Respir Crit Care Med 2010;181:1391-6.

51. Ettinger NA, Bailey TC, Trulock EP, et al. Cytomegalovirus infection and pneumonitis. Impact after isolated lung transplantation. Washington University Lung Transplant Group. Am Rev Respir Dis 1993;147:1017-23.

52. Desai R, Collett D, Watson CJ, et al. Impact of Cytomegalovirus on Long-term Mortality and Cancer Risk After Organ Transplantation. Transplantation 2015;99:1989-94.

53. Zamora MR, Nicolls MR, Hodges TN, et al. Following universal prophylaxis with intravenous ganciclovir and cytomegalovirus immune globulin, valganciclovir is safe and effective for prevention of CMV infection following lung transplantation. Am J Transplant 2004;4:1635-42.

54. Jaksch P, Zweytick B, Kerschner H, et al. Cytomegalovirus prevention in high-risk lung transplant recipients: comparison of 3-vs 12 -month valganciclovir therapy. J Heart Lung Transplant 2009;28:670-5.

55. Palmer SM, Limaye AP, Banks M, et al. Extended valganciclovir prophylaxis to prevent cytomegalovirus after lung transplantation: a randomized, controlled trial. Ann Intern Med 2010;152:761-9.

56. Marty FM, Ljungman P, Chemaly RF, et al. Letermovir Prophylaxis for Cytomegalovirus in Hematopoietic-Cell Transplantation. N Engl J Med 2017;377:2433-44. 
57. Kruger RM, Paranjothi S, Storch GA, et al. Impact of prophylaxis with cytogam alone on the incidence of CMV viremia in $\mathrm{CMV}$-seropositive lung transplant recipients. J Heart Lung Transplant 2003;22:754-63.

58. Le Page AK, Jager MM, Kotton CN, et al. International survey of cytomegalovirus management in solid organ transplantation after the publication of consensus guidelines. Transplantation 2013;95:1455-60.

59. Kotton CN, Kumar D, Caliendo AM, et al. The Third International Consensus Guidelines on the Management of Cytomegalovirus in Solid-organ Transplantation. Transplantation 2018;102:900-31.

60. Asberg A, Jardine AG, Bignamini AA, et al. Effects of the intensity of immunosuppressive therapy on outcome of treatment for CMV disease in organ transplant recipients. Am J Transplant 2010;10:1881-8.

61. Bhorade SM, Lurain NS, Jordan A, et al. Emergence of ganciclovir-resistant cytomegalovirus in lung transplant recipients. J Heart Lung Transplant 2002;21:1274-82.

62. Fisher CE, Knudsen JL, Lease ED, et al. Risk Factors and Outcomes of Ganciclovir-Resistant Cytomegalovirus Infection in Solid Organ Transplant Recipients. Clin Infect Dis 2017;65:57-63.

63. Drew WL, Miner RC, Marousek GI, et al. Maribavir sensitivity of cytomegalovirus isolates resistant to ganciclovir, cidofovir or foscarnet. J Clin Virol 2006;37:124-7.

64. Papanicolaou GA, Silveira FP, Langston AA, et al. Maribavir for Refractory or Resistant Cytomegalovirus Infections in Hematopoietic-cell or Solid-organ Transplant Recipients: A Randomized, Dose-ranging, Double-blind, Phase 2 Study. Clin Infect Dis 2019;68:1255-64.

65. Yao QY, Rickinson AB, Epstein MA. A re-examination of the Epstein-Barr virus carrier state in healthy seropositive individuals. Int J Cancer 1985;35:35-42.

66. Taylor GS, Long HM, Brooks JM, et al. The immunology of Epstein-Barr virus-induced disease. Annu Rev Immunol 2015;33:787-821.

67. Sebelin-Wulf K, Nguyen TD, Oertel S, et al. Quantitative analysis of EBV-specific CD4/CD8 T cell numbers, absolute CD4/CD8 $\mathrm{T}$ cell numbers and EBV load in solid organ transplant recipients with PLTD. Transpl Immunol 2007;17:203-10.

68. Falco DA, Nepomuceno RR, Krams SM, et al. Identification of Epstein-Barr virus-specific CD8+ T lymphocytes in the circulation of pediatric transplant recipients. Transplantation 2002;74:501-10.

69. Hatton OL, Harris-Arnold A, Schaffert S, et al. The interplay between Epstein-Barr virus and B lymphocytes: implications for infection, immunity, and disease. Immunol Res 2014;58:268-76.

70. Allen U, Preiksaitis J. Epstein-barr virus and posttransplant lymphoproliferative disorder in solid organ transplant recipients. Am J Transplant 2009;9 Suppl 4:S87-96.

71. Dharnidharka VR, Webster AC, Martinez OM, et al. Posttransplant lymphoproliferative disorders. Nat Rev Dis Primers 2016;2:15088.

72. Kumarasinghe G, Lavee O, Parker A, et al. Posttransplant lymphoproliferative disease in heart and lung transplantation: Defining risk and prognostic factors. J Heart Lung Transplant 2015;34:1406-14.

73. Evens AM, Roy R, Sterrenberg D, et al. Posttransplantation lymphoproliferative disorders: diagnosis, prognosis, and current approaches to therapy. Curr Oncol Rep 2010;12:383-94.

74. Kremer BE, Reshef R, Misleh JG, et al. Post-transplant lymphoproliferative disorder after lung transplantation: a review of 35 cases. J Heart Lung Transplant 2012;31:296-304.

75. Reams BD, McAdams HP, Howell DN, et al. Posttransplant lymphoproliferative disorder: incidence, presentation, and response to treatment in lung transplant recipients. Chest 2003;124:1242-9.

76. Prockop SE, Vatsayan A. Epstein-Barr virus lymphoproliferative disease after solid organ transplantation. Cytotherapy 2017;19:1270-83.

77. Al-Mansour Z, Nelson BP, Evens AM. Post-transplant lymphoproliferative disease (PTLD): risk factors, diagnosis, and current treatment strategies. Curr Hematol Malig Rep 2013;8:173-83.

78. Glotz D, Chapman JR, Dharnidharka VR, et al. The Seville expert workshop for progress in posttransplant lymphoproliferative disorders. Transplantation 2012;94:784-93.

79. Wheless SA, Gulley ML, Raab-Traub N, et al. Posttransplantation lymphoproliferative disease: Epstein-Barr virus DNA levels, HLA-A3, and survival. Am J Respir Crit Care Med 2008;178:1060-5.

80. Blaes AH, Peterson BA, Bartlett N, et al. Rituximab therapy is effective for posttransplant lymphoproliferative disorders after solid organ transplantation: results of a phase II trial. Cancer 2005;104:1661-7.

81. Trappe R, Oertel S, Leblond V, et al. Sequential treatment with rituximab followed by $\mathrm{CHOP}$ chemotherapy in adult B-cell post-transplant lymphoproliferative disorder (PTLD): the prospective international multicentre phase 2 
PTLD-1 trial. Lancet Oncol 2012;13:196-206.

82. Dierickx D, Tousseyn T, Gheysens O. How I treat posttransplant lymphoproliferative disorders. Blood 2015;126:2274-83.

83. Haque T, Wilkie GM, Jones MM, et al. Allogeneic cytotoxic T-cell therapy for EBV-positive posttransplantation lymphoproliferative disease: results of a phase 2 multicenter clinical trial. Blood 2007;110:1123-31.

84. Fryer JF, Heath AB, Wilkinson DE, et al. A collaborative study to establish the 1st WHO International Standard for Epstein-Barr virus for nucleic acid amplification techniques. Biologicals 2016;44:423-33.

85. Wagner HJ, Wessel M, Jabs W, et al. Patients at risk for development of posttransplant lymphoproliferative disorder: plasma versus peripheral blood mononuclear cells as material for quantification of Epstein-Barr viral load by using real-time quantitative polymerase chain reaction. Transplantation 2001;72:1012-9.

86. McDiarmid SV, Jordan S, Kim GS, et al. Prevention and preemptive therapy of postransplant lymphoproliferative disease in pediatric liver recipients. Transplantation 1998;66:1604-11.

87. Kobayashi S, Sano H, Mochizuki K, et al. Pre-emptive rituximab for Epstein-Barr virus reactivation after haplohematopoietic stem cell transplantation. Pediatr Int 2017;59:973-8.

88. Xu F, Schillinger JA, Sternberg MR, et al. Seroprevalence and coinfection with herpes simplex virus type 1 and type 2 in the United States, 1988-1994. J Infect Dis 2002;185:1019-24.

89. Smyth RL, Higenbottam TW, Scott JP, et al. Herpes simplex virus infection in heart-lung transplant recipients. Transplantation 1990;49:735-9.

90. Wilck MB, Zuckerman RA. Herpes simplex virus in solid organ transplantation. Am J Transplant 2013;13 Suppl 4:121-7.

91. Pham MA, Bednarczyk RA, Becker ER, et al. Trends in U.S. Community hospitalizations due to herpes zoster: 2001-2015. Vaccine 2019;37:882-8.

92. Manuel O, Kumar D, Singer LG, et al. Incidence and clinical characteristics of herpes zoster after lung transplantation. J Heart Lung Transplant 2008;27:11-6.

93. Pergam SA, Limaye AP. Varicella Zoster Virus (VZV) in Solid Organ Transplantation: Guidelines from the American Society of Transplantation Infectious Diseases Community of Practice. Clin Transplant 2019:e13622.
94. Danziger-Isakov L, Kumar D. Vaccination of solid organ transplant candidates and recipients: Guidelines from the American society of transplantation infectious diseases community of practice. Clin Transplant 2019:e13563.

95. Cunningham AL, Heineman T. Vaccine profile of herpes zoster (HZ/su) subunit vaccine. Expert Rev Vaccines 2017;16:1-10.

96. Levitsky J, Doucette K. Viral hepatitis in solid organ transplantation. Am J Transplant 2013;13 Suppl 4:147-68.

97. Poland GA, Jacobson RM. Clinical practice: prevention of hepatitis B with the hepatitis B vaccine. N Engl J Med 2004;351:2832-8.

98. Weill D, Benden C, Corris PA, et al. A consensus document for the selection of lung transplant candidates: 2014--an update from the Pulmonary Transplantation Council of the International Society for Heart and Lung Transplantation. J Heart Lung Transplant 2015;34:1-15.

99. Lauer GM, Walker BD. Hepatitis C virus infection. N Engl J Med 2001;345:41-52.

100. Morales JM, Campistol JM. Transplantation in the patient with hepatitis C. J Am Soc Nephrol 2000;11:1343-53.

101.Fong TL, Cho YW, Hou L, et al. Outcomes after lung transplantation and practices of lung transplant programs in the United States regarding hepatitis C seropositive recipients. Transplantation 2011;91:1293-6.

102. Chung RT, Feng S, Delmonico FL. Approach to the management of allograft recipients following the detection of hepatitis B virus in the prospective organ donor. Am J Transplant 2001;1:185-91.

103. Nangia G, Borges K, Reddy KR. Use of HCV-infected organs in solid organ transplantation: An ethical challenge but plausible option. J Viral Hepat 2019;26:1362-71.

104. Watson J, Mulvihill MS, Cox ML, et al. Early experience with the use of hepatitis $C$ antibody-positive, nucleic acid testing-negative donors in lung transplantation. Clin Transplant 2019;33:e13476.

105. Schlendorf KH, Zalawadiya S, Shah AS, et al. Early outcomes using hepatitis C-positive donors for cardiac transplantation in the era of effective direct-acting antiviral therapies. J Heart Lung Transplant 2018;37:763-9.

Cite this article as: Clausen ES, Zaffiri L. Infection prophylaxis and management of viral infection. Ann Transl Med 2020;8(6):415. doi: 10.21037/atm.2019.11.85 